\title{
Lipase-catalyzed Reactions at Interfaces of Two-phase Systems and Microemulsions
}

\author{
P. Reis $・$ R. Miller $・$ M. Leser $\cdot$ H. Watzke
}

Received: 30 June 2008 / Accepted: 19 August 2008 /

Published online: 16 September 2008

(C) Humana Press 2008

\begin{abstract}
This work describes the influence of two polar lipids, Sn-1/3 and Sn-2 monopalmitin, on the activity of lipase in biphasic systems and in microemulsions. In previous communications, we have shown that $\mathrm{Sn}-2$ monoglycerides can replace $\mathrm{Sn}-1,3$ regiospecific lipases at the oil-water interface, causing a drastically reduced rate of lipolysis. We here demonstrate that even if the lipase is expelled from the interface, it can catalyze esterification of the Sn-2 monoglyceride with fatty acids in both macroscopic oilwater systems and in microemulsions, leading to formation of di- and triglycerides.
\end{abstract}

Keywords Lipase $\cdot$ Esterification $\cdot$ Synthesis $\cdot$ Interface

\section{Introduction}

Lipases are versatile enzymes that catalyze not only hydrolysis but also synthesis of acylglycerides [1]. They are active at the oil-water interface of emulsified substrates [2], where the interfacial composition has been found to change continuously during the biocatalysis [3].

The "quality of the interface" is known to be an essential parameter for lipase activity [4]. There is no commonly accepted definition for this term [5]. However, it is thought to be influenced by interfacial tension and its structure and composition. It is known that too low surface pressure, i.e., too high interfacial tension, may lead to irreversible lipase

P. Reis $\cdot$ M. Leser $\cdot$ H. Watzke

Nestlé Research Center, 1000 Lausanne 26, Switzerland

P. Reis $(\bowtie)$

Department of Chemical and Biological Engineering, Applied Surface Chemistry,

Chalmers University of Technology, 41296 Göteborg, Sweden

e-mail: pedro_esb@hotmail.com

R. Miller

Max-Planck-Institut für Kolloid- und Grenzflächenforschung, Max-Planck-Campus, 14476 Golm, Germany 
denaturation [6]. It has also been claimed that very high surface pressure gives a decrease in lipase activity as a result of inaccessibility of the substrate to the active site of the lipase [7]. However, when comparing the inhibition effect obtained with different amphiphiles with the effect on the interfacial tension of a triolein/water interface, one can conclude that there is no direct correlation between the decrease in lipase activity and the lowering of the interfacial tension between the aqueous and the oil phase [8]. Furthermore, lipase catalysis occurs readily in microemulsions, which have extremely low internal oil-water interfacial tensions, i.e., high surface pressures [9]. The value of interfacial tension per se is evidently not decisive of the activity of the enzyme. Moreover, the activity of a lipase at an interface may be affected by charged amphiphiles also present at the interface. Fatty acid salts may lead to enzyme inhibition as a result of conformational changes caused by electrostatic attraction between the anionic headgroup of the alkyl carboxylate and sites of opposite charge on the enzyme [10]. The adsorption from mixed protein/surfactant solutions can be studied generally via two different experimental routes, i.e., via sequential and simultaneous adsorption [11-14]. In both cases, mixed layers are formed, the properties of which have to be measured and quantitatively understood in order to have direct influence on the quality of products. Therefore, the effect of the interfacial properties (e.g., tension/pressure) on lipase activity can be further explored. Progress was made in recent times due to modern experimental techniques available for systematic studies, such as tensiometry [15], ellipsometry [16], Brewster angle microscopy [11], interfacial dilational [17], and shear rheology [18]. In addition, quantitative theoretical models have been derived for describing the equilibrium state, as well as the dynamics and dilational rheology for mixed adsorption layers. Thus, the changes in interface tension, equilibrium and dynamic adsorption, and 2D rheological characteristics due to the addition of surfactants to protein layers at liquid/fluid interfaces can be described at least semiquantitatively [17, 19-25].

It is also known that lipases, like all other enzymes, may be subject to competitive inhibition [26] or to inhibition due to formation of complexes that alter the conformation of the enzyme [27]. It is evident that a number of issues remain to be clarified before the term "quality of the interface" becomes a useful predictive tool.

This work aims at providing information about how highly interfacially active amphiphiles present at the oil-water interface will affect the activity of the lipase. More specifically, we investigate how the presence of a Sn-2 monoglyceride, which expels the lipase from the interface, affects the ability of the lipase to catalyze hydrolysis and esterification reactions with lipophilic substrates.

\section{Materials and Methods}

\section{Chemicals}

Lipase from Rhizomucor miehei (4,210 units/mg solid), lipoprotein lipase from Chromobacterium viscosum (2,500 units/mg solid), lipase from porcine pancreas (100-400 units/mg solid), and lipase from Candida rugosa (10,699 units/mg solid) were obtained from Sigma, St Louis, MO, USA. The lipase preparations from $R$. miehei and C. rugosa and lipoprotein lipase from $C$. viscosum are rather pure but contain traces of other compounds (propanediol, potassium sorbate, sodium benzoate, sorbitol) that are used to stabilize the liophylized form of the enzyme. The lipase from porcine pancreas is a crude preparation that contains other substances present in the duodenum (bile, proteases, colipase, etc.). 4-Nitrophenylpalmitate (p-nitrophenylpalmitate, pNPP), hexadecyltrimethylammonium bromide (99\%), decane 
(>95.0\%), and tert-butanol (>99.5\%) were obtained from Sigma. Tricaprylin (99\%), dicaprylin (99\%), Sn-2 monocaprylin (99\%), tripalmitin (99\%), dipalmitin (99\%), Sn-2 monopalmitin (99\%), and lauric acid (99\%) were obtained from Larodan, Malmö, Sweden. Sn-2 mono-cis-4,7,10,13,16,19-docosahexaenoin (monoarachidin) was from Nu-Check Prep, Elysian, MN, USA.

\section{Pendant Drop Technique}

Interfacial tension was recorded on a tensiometer from Teclis/IT Concept. A syringe of $250 \mu \mathrm{l}$ from Hamilton 1001 TLLWS was used with a needle $1.83 \mathrm{~mm}$ in diameter. If not otherwise mentioned, the aqueous phase was held in the syringe, while the apolar phase was kept in a HEL-101-OS-20T cell from Hellma. The principle of the drop profile analysis is based on determination of the coordinates of a liquid drop from a video image and comparing these coordinates with theoretical profiles calculated from the Gauss-Laplace equation [28]. All the measurements were performed using the area mode control of an aqueous drop of $2.97 \mathrm{~mm}^{3}$.

\section{GC-FID}

Glycerides were analyzed using an Agilent 6890 gas chromatograph equipped with a flame ionization detection system. If not otherwise mentioned, $100 \mu \mathrm{l}$ of either the apolar phase of a biphasic system or a microemulsion were added into $900 \mu \mathrm{l}$ of chloroform before injection. Separation of the surface active molecules was carried out in a nonpolar fused silica column (30 $\mathrm{m}$ long with an inner diameter of $0.25 \mathrm{~mm}$ ) coated with methylphenylpolysiloxane (DB-5 HT from J\&W). Sample injection $(1 \mu \mathrm{l})$ was performed directly on the column with the injector tracking oven temperature. The initial oven temperature was set to $90^{\circ} \mathrm{C}$ for $2 \mathrm{~min}$, followed by an increase of $10^{\circ} \mathrm{C} / \mathrm{min}$ for $8 \mathrm{~min}$. The detector temperature was kept constant at $360^{\circ} \mathrm{C}$. Recording of chromatograms was performed with an HP-Chemstation for GC Systems (Software Chemstation, version A10.01). Chromatographic peaks were identified by comparing the retention times with those of known standards.

\section{Liquid Chromatography-Mass Spectrometry Analysis}

Liquid chromatography-mass spectrometry (LC-MS) analyses were carried out using a triple quadrupole mass spectrometer (Applied Biosystems API 4000) coupled to a high-performance liquid chromatography (HPLC) system (Agilent 1100). During operation, the atmospheric pressure chemical ionization probe temperature was set to $450{ }^{\circ} \mathrm{C}$ and the current (NC) to $4 \mu \mathrm{A}$. Nitrogen was used for the nebulizer, the ion source gas $(=30)$, and the cone gas curtain $(=15)$. Mass spectra were acquired by scanning over the mass range $m / z 150$ to 700 with a scan time of $1 \mathrm{~s}$, and with a declustering potential of $60 \mathrm{~V}$. Five microliters was injected into the HPLC column. A C18 column $(150 \times 3.0 \mathrm{~mm}, 3.5 \mu \mathrm{m}$ particle size, Sunfire, Waters, Milford, USA) was used with acetonitrile (solvent A) and chloroform (solvent B) at a flow rate of $1.0 \mathrm{ml} / \mathrm{min}$. The gradient started at $100 \% \mathrm{~A}$ and was held constant for $3 \mathrm{~min}$, followed by a linear increase to $80 \% \mathrm{~A}$ at $10 \mathrm{~min}$. Solvent composition returned to initial conditions at $12 \mathrm{~min}$. The next injection was performed $3 \mathrm{~min}$ after reestablishing the initial gradient conditions.

\section{Spectrophotometry}

A Hp-8452 UV-visible spectrophotometer was used to follow lipase hydrolysis of 4nitrophenylpalmitate by measuring the absorption of nitrophenol at $405 \mathrm{~nm}$ at room temperature. 
Dispersions Stability Test

A TurbiScan Lab from Formulaction with a pulsed near infrared LED light source at $880 \mathrm{~nm}$ was used in the transmission mode to assess the stability of dispersions. A cylindrical glass measurement cell with $55 \mathrm{~mm}$ length was adopted to perform the measurements. Different transmission profiles are expected in the sample if phase separation occurs.

\section{Results and Discussion}

\section{Biphasic Systems}

Figure 1 shows the effect of different monoglycerides, added in a fixed concentration, on hydrolysis of 4-nitrophenylpalmitate (pNPP) in a buffer/decane biphasic system containing lipase from $R$. miehei. The hydrolysis of $\mathrm{pNPP}$ is monitored by measuring the concentration of one of the products of the lipolysis, 4-nitrophenol, in the aqueous phase by spectrophotometry at $405 \mathrm{~nm}$. The results show that Sn-2 monocaprylin and, even more, Sn-2 monopalmitin decrease the rate of pNPP hydrolysis. The long-chain and polyunsaturated Sn-2 monoarachidin did not affect the reaction rate. However, when used in 10 times higher concentration, the latter Sn-2 monoglyceride also effectively prevented hydrolysis of pNPP during a reaction time of $8 \mathrm{~h}$ (data not shown).

As can be seen from Fig. 1, the Sn-1/3 monoglycerides, which are known to be rapidly cleaved by the $\mathrm{Sn}-1,3$ regiospecific lipase, gave the same reaction profile as the system without added monoglyceride. A reasonable explanation of this behavior is that the $S n-1 / 3$ monoglyceride, but not the $S n-2$ monoglyceride, is rapidly hydrolyzed to the less interfacially active degradation products fatty acid and glycerol, which cannot compete with the lipase for a place at the interface [29].

Monoglycerides are highly surface-active molecules. In a recent work, we have shown that Sn-2 monocaprylin and Sn-2 monopalmitin reduce the interfacial tension of the decane/ buffer system from $46 \mathrm{mN} / \mathrm{m}$ to 6 and $10 \mathrm{mN} / \mathrm{m}$, respectively [30]. A monoglyceride can therefore be expected to expel the much less surface-active pNPP $(44 \mathrm{mN} / \mathrm{m})$ from the interface already at very low concentration. Thus, it is not unexpected that pNPP will remain intact as long as there is monoglyceride at the oil-water interface.

Fig. 1 Effect of addition of $1.3 \mathrm{mM} \mathrm{Sn}-1 / 3$ monocaprylin (Sn-1/3 MC8), Sn-2 monocaprylin (Sn-2 MC8), Sn-1/3 monopalmitin (Sn-1/3 MC16), Sn-2 monopalmitin (Sn-2 MC16), or $\mathrm{Sn}-2$ monoarachidin (Sn-2 MC20:4) on the generation of 4-nitrophenol due to hydrolysis of 6.6 E-4M 4-nitrophenylpalmitate in the biphasic system $7.0 \mathrm{ml}$ decane/ $2.0 \mathrm{ml} 0.1 \mathrm{M}$ phosphate buffer $\mathrm{pH} 7.0$, with an interface of $82 \mathrm{~mm}^{2}$ and with $3.3 \mathrm{E}-5 \mathrm{M}$ lipase from $R$. miehei added to the aqueous phase. A control with no monoglyceride (No $M G$ ) was also performed

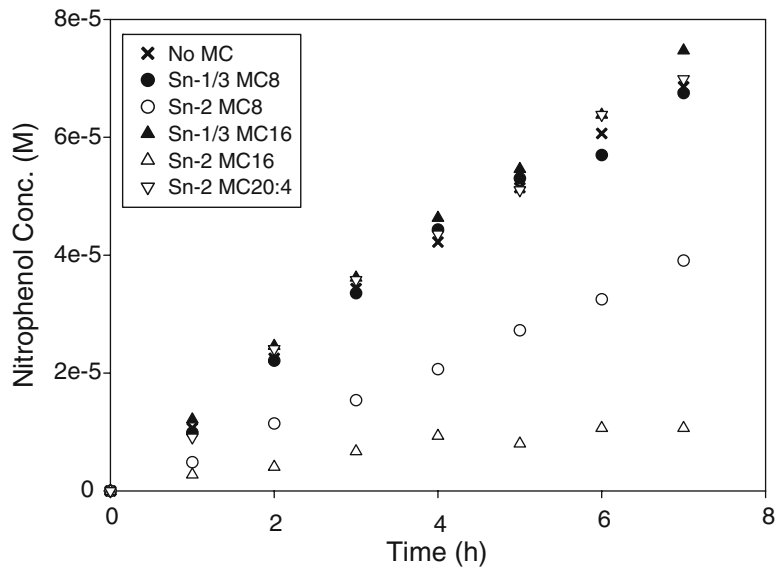


It is likely that the relative efficiency of the Sn-2 monoglycerides in preventing lipasecatalyzed hydrolysis of pNPP, i.e., monopalmitin $>$ monocaprylin $>$ monoarachidin, is due to differences in the partitioning and interfacial activity of these amphiphiles [30]. Monopalmitin is a well balanced amphiphile in the decane-water system. Its solubility in water is almost negligible. It partitions between the interface and the oil phase but the oil solubility is not exceedingly high. The interface can be regarded as efficiently covered by the monoglyceride.

Monocaprylin is also a balanced amphiphile with a strong driving force for the interface. However, it has relatively high solubility in the aqueous phase. It will form micelles in the water phase, and these micelles will contain solubilized oil. Thus, the water phase can be said to be an oil-in-water microemulsion. The solubilized oil is likely to have the same composition as the macroscopic oil phase, i.e., it will consist of decane with dissolved pNPP. However, acyl migration occurs faster for Sn-2 monocaprylin than for Sn-2 monopalmitin (data not shown). The lipase and the noncleavable monocaprylin isomer will compete for a place at the micelle-water interface just as they compete for a place at the macroscopic oil-water interface. The monoglyceride will be the favored amphiphile at both interfaces, but due to the fact that the interface will be greatly enlarged by the formation of oil-swollen micelles, the probability of pNNP reaching the lipase active site will increase compared to the situation with monopalmitin.

Monoarachidin, having a C20 acyl chain, is a very lipophilic amphiphile. It partitions strongly into the oil phase, and its driving force for the interface is not as strong as for the other, more balanced monoglycerides. Thus, monoarachidin does not prevent the lipase and its substrate, pNPP, to meet and react at the interface.

We have also previously shown that lipase from $R$. miehei, which is Sn-1,3 regiospecific, can be displaced from the interface by balanced Sn-2 monoglycerides, such as Sn-2 monopalmitin and Sn-2 monocaprylin [3, 31]. Complete removal of the enzyme from the buffer/decane interface was obtained at very low bulk concentration of these monoglycerides. However, for Sn-2 monoarachidin, a higher concentration was required. Figure 2 shows the effect on the interfacial tension obtained by injecting Sn-2 monocaprylin, Sn-2 monopalmitin, and Sn-2 monoarachidin at a concentration of $5.0 \mathrm{mM}$, and also the latter monoglyceride at a concentration of $15.3 \mathrm{mM}$.

Figure 2a also shows the effect of addition of Sn-1/3 monocaprylin and Sn-1/3 monopalmitin. These monoglycerides are substrates for the 1,3-specific lipase, and the interfacial tension rapidly reverts to the value given by lipase at the interface. The recovery of the interfacial tension upon lipase hydrolysis of the Sn-1/3 monoglycerides will depend both on lipase affinity for the substrates and on the partitioning of the reaction products from the interface into the bulk phases.

Many proteins are expelled from the interface in the presence of low-molecular-weight surfactants that increase the interfacial pressure. With tensiometry, one follows the change in surface free energy but the technique does not per se give information about what molecules are present at the interface. Interfacial shear rheology gives complementary information because it provides information on the stiffness of the interface. The kinetic irreversibility of protein adsorption in pure systems normally leads to the formation of very stiff layers, while the reversibility of low-molecular-weight surfactants adsorption usually leads to flexible ones. The torsion pendulum technique gives information about the stiffness of the interfacial layer. By measuring the variation of oscillation amplitude with time, a change in rheological properties of this layer can be detected. Previous results have confirmed that Sn-2 monoglycerides exclude the lipase from the interface as the rheological properties of the mixed system correspond to the one of the low-molecular-weight 
Fig. 2 Interfacial tension of a buffer/decane system with 3.3 E-5 M lipase from $R$. miehei added followed by injection into the oil phase of monoglycerides $(M G)$ 1,000 s after drop formation. a $5.0 \mathrm{mM} \mathrm{Sn}-2$ monocaprylin (Sn-2 MC8), Sn-1/3 monocaprylin (Sn-1/3 MC8), Sn-2 monopalmitin (Sn-2 MC16), or Sn-1/3 monopalmitin (Sn-1/3 MC16); b $5.0 \mathrm{mM}$ or $15.3 \mathrm{mM} \mathrm{Sn}-2$ monoarachidin (Sn-2 MC20:4)
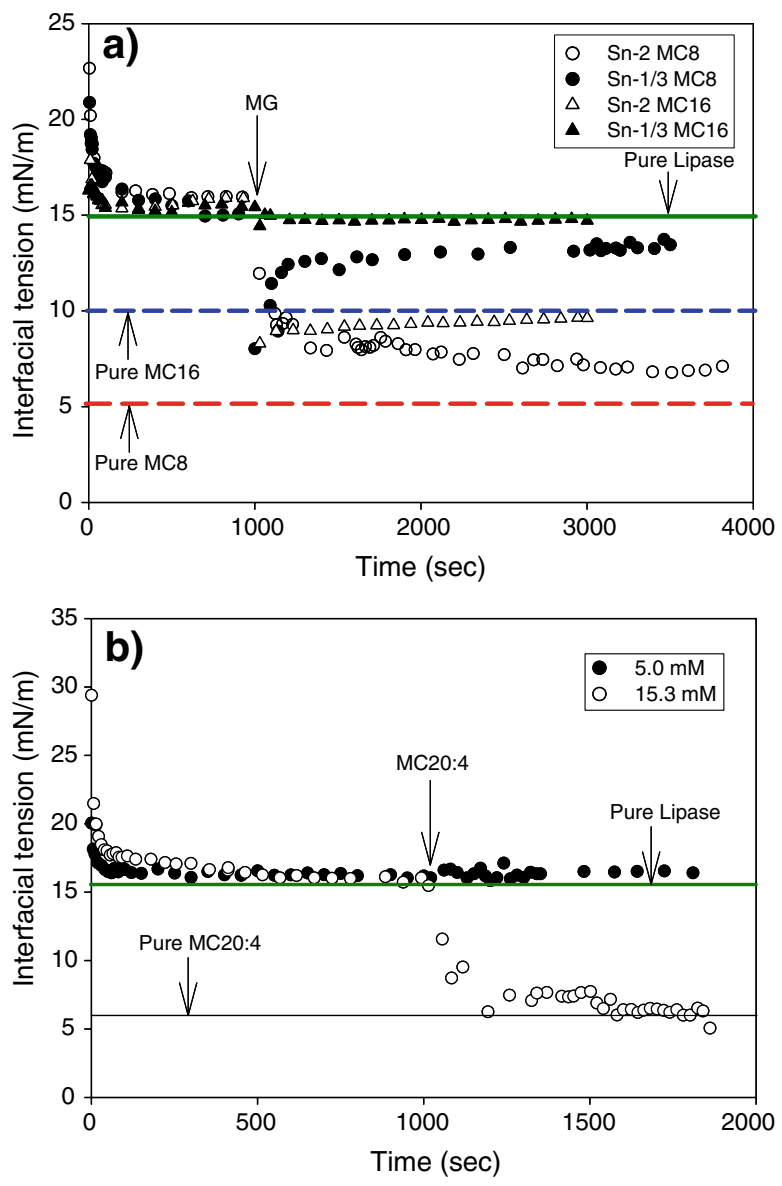

surfactant [29]. Triglyceride hydrolysis catalyzed by a $S n-1,3$ regiospecific lipase will yield $S n-2$ monoglyceride and fatty acid, while hydrolysis catalyzed by a nonregiospecific lipase will give fatty acid and glycerol. Thus, if lipolysis occurs with a $S n-1,3$ regiospecific lipase, but not with a nonspecific lipase, a reaction product will form that is so interfacially active that the lipase and its substrate are expelled from the interface.

Removal of the lipase from the interface by the more interfacially active Sn-2 monoglyceride was found to prevent further degradation of a triglyceride present in the oil phase. Also, in an in vitro model of the gastro-intestinal system, the presence of Sn-2 monoglyceride caused retardation of lipolysis [32]. We have postulated that the generation of Sn-2 monoglycerides at the oil-water interface is a self-regulatory process that eventually makes fat digestion come to a stop [31, 32].

In order to evaluate if lipase is entirely inactivated when a highly interfacially active Sn-2 monoglyceride is present at the interface of the buffer/decane system, lauric acid, tricaprylin, and Sn-2 monopalmitin were added to the oil phase and lipase from $R$. miehei was added to the aqueous phase. The oil phase was analyzed by LC-MS after $75 \mathrm{~h}$ reaction time, Fig. 3. It can clearly be seen that monopalmitin has almost vanished and that a wide variety of di- and triglycerides have been generated. The results indicate that the Sn-2 monoglyceride has undergone lipase-catalyzed esterification. 


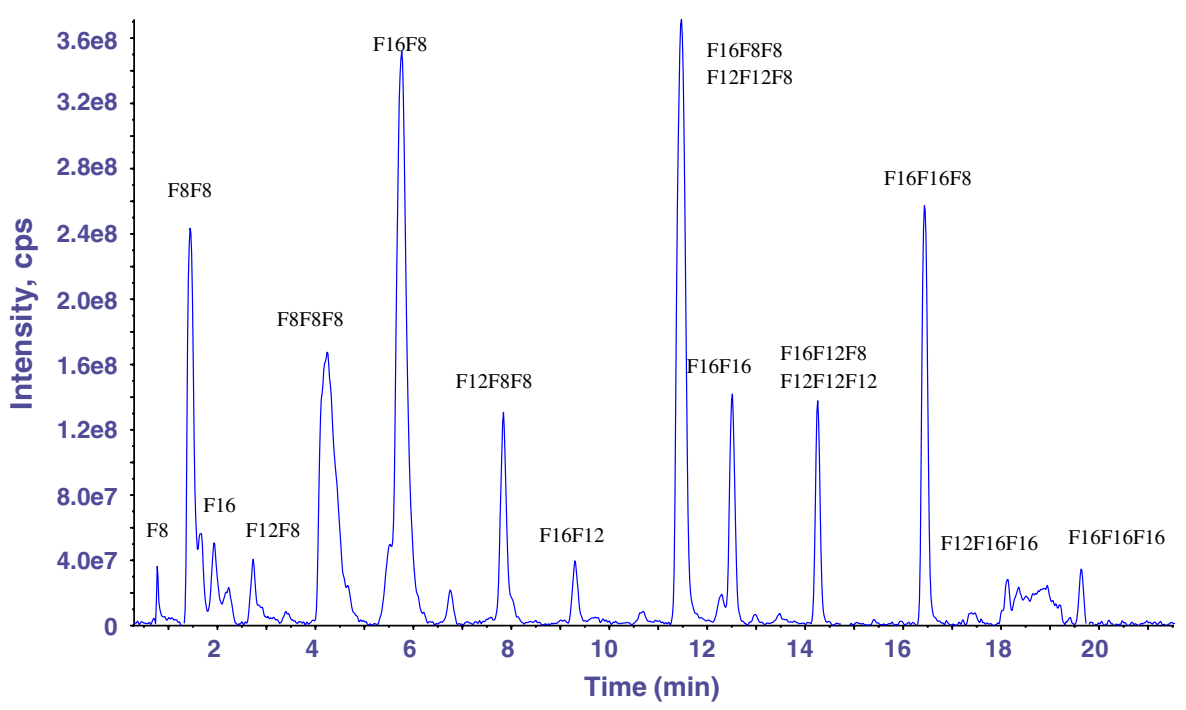

Fig. 3 LC-MS spectrum of the apolar phase after $75 \mathrm{~h}$ reaction at $25^{\circ} \mathrm{C}$ in the biphasic system consisting of $7.0 \mathrm{ml}$ decane containing $4.5 \mathrm{mM}$ lauric acid, 8.4 E-3 M tricaprylin, and 4.1 E-3 M Sn-2 monopalmitin and a water phase consisting of $2.0 \mathrm{ml} 0.1 \mathrm{M}$ phosphate buffer $\mathrm{pH} 7.0$ with $3.3 \mathrm{E}-6 \mathrm{M}$ lipase from $R$. miehei added. $F$ monoglyceride, $F F$ diglyceride, $F F F$ triglyceride

The same experiment was also made with the lipase from $R$. miehei replaced by lipoprotein lipase. This is an enzyme widely found in endothelial cells. It hydrolyzes lipids in lipoproteins, such as those present in chylomicrons and very low density lipoproteins [33]. Like lipase from $R$. miehei, it exhibits Sn-1,3 regiospecificity. The result obtained with this lipase with respect to peaks in the GC-FID chromatogram of the oil phase after completed reaction was very similar to that obtained with lipase $R$. miehei (data not shown).

As mentioned above, we have previously found that a Sn-2 monoglyceride pushes the lipase away from the interface into the water phase, effectively preventing further lipolysis of triglycerides present in the apolar phase. In fact, we have shown by a combination of ellipsometry and surface rheology that the lipase seems to be present in a sublayer just below the interface [29]. Based on this knowledge, we believe that a likely scenario for the biocatalytic events leading to the product mixture shown in Fig. 3 is the following: The lipase catalyzes esterification of the monopalmitin with the added lauric acid, which partitions between the oil phase (in the form of protonated fatty acid) and the water phase (as the salt). Both lauric acid and sodium laurate are less interfacially active than either Sn-2 monopalmitin or lipase [30]; thus, neither of them will be present at the interface. It is likely that the reaction occurs in the aqueous sublayer with the fatty acid salt as substrate. The diglyceride formed is lipophilic and will partition into the oil phase. Eventually, the monoglyceride will have disappeared from the interface and the second substance in terms of interfacial activity, the lipase, will have taken its place. The lipase is now able to catalyze all sorts of esterification and transesterification reactions, which leads to a wide spectrum of di- and triglycerides, including acylglycerides containing capryl (octanoyl) chains, originating from the added tricaprylin.

Bile salts are known to be good emulsifiers for lipids and, in particular, effective solubilizers of fatty acids [34, 35]. The enlargement of the interface and the presence of a new interfacially active component may influence the biocatalysis at the interface. The fatty 
acid solubilization capacity might also affect the lipolysis pattern since fatty acid formed during the lipolysis could be removed from the reaction zone. Figure 4 shows that bile salts reduce the amount of Sn-2 monopalmitin in the oil phase of the (lipase-free) buffer/decane system by more than $90 \%$. Most likely, this is due to solubilization of the monoglyceride into the aqueous phase. In in vivo systems, the in situ generated Sn-2 monoglycerides are solubilized by bile salts from the interface of water/oil drops and transported across the brush border cells of the gastro-intestinal system. This delivery mechanism provides a reestablishment of the solubility capacity of bile salts and a high yield of fat absorption. Since Sn-2 monopalmitin is almost quantitatively removed from the oil phase by bile salts, one may anticipate that the oil-water interface of a biphasic system will not be fully covered by the monoglyceride that is so efficient in expelling the lipase. The lipase is then likely to dominate the interface. This, in turn, would mean that there will be no, or little, inhibiting effect of a Sn-2 monoglyceride on the catalytic activity of a Sn-1,3 regiospecific lipase if a bile salt is present in the system.

Figure 5 demonstrates that this is indeed the case. Figure 5a shows the composition of the oil phase of the buffer/decane system with tricaprylin added to the decane and phosphate buffer with pancreatic lipase, which is Sn-1,3 regiospecific. After $72 \mathrm{~h}$ of reaction time, $48 \%$ of tricaprylin hydrolysis has occurred. Figure $5 \mathrm{c}$ shows what happens when Sn-2 monopalmitin is added to the decane phase in a system with pancreatic lipase and no bile salts. A quantitative analysis based on the GC peaks showed that $27 \%$ of the tricaprylin had hydrolyzed after $72 \mathrm{~h}$ at $25^{\circ} \mathrm{C}$. Two new peaks with longer retention times than that of tricaprylin can be seen. It is likely (but not verified) that the peak close to $6 \mathrm{~min}$ represents the diglyceride having palmitic acid in Sn-2 position and caprylic acid in Sn-1/3 position and the peak close to $7 \mathrm{~min}$ represents the triglyceride with palmitic acid in the
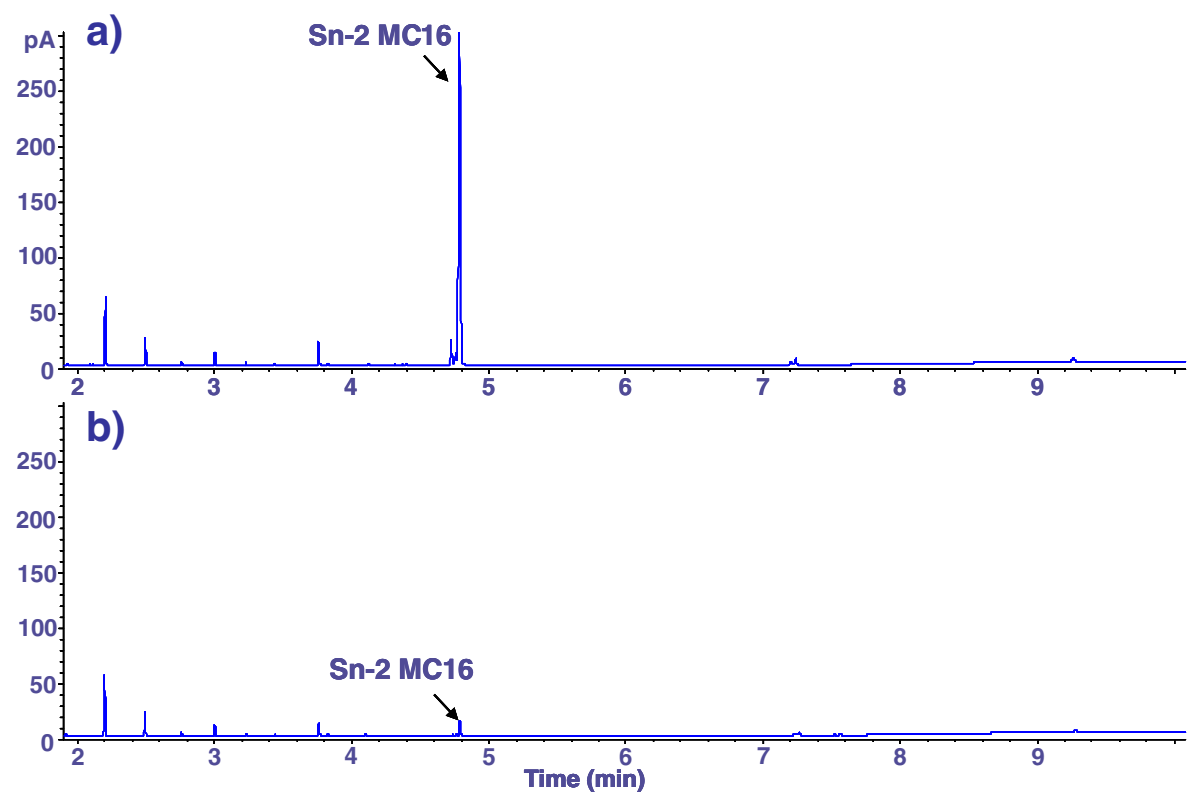

Fig. 4 GC-FID chromatogram of the oil phase from a biphasic system consisting of $3.0 \mathrm{ml}$ decane containing $8.3 \mathrm{mM} \mathrm{Sn}-2$ monopalmitin $(\mathrm{Sn}-2 \mathrm{MCl})$ and $5 \mathrm{ml}$ phosphate buffer a without and $\mathbf{b}$ with bile salts $(0.4 \mathrm{wt} . \%)$ added. Both systems were vortexed for $1 \mathrm{~min}$ and centrifuged during $5 \mathrm{~min}$ at $4,000 \mathrm{rpm}$ before separation of the apolar phase 


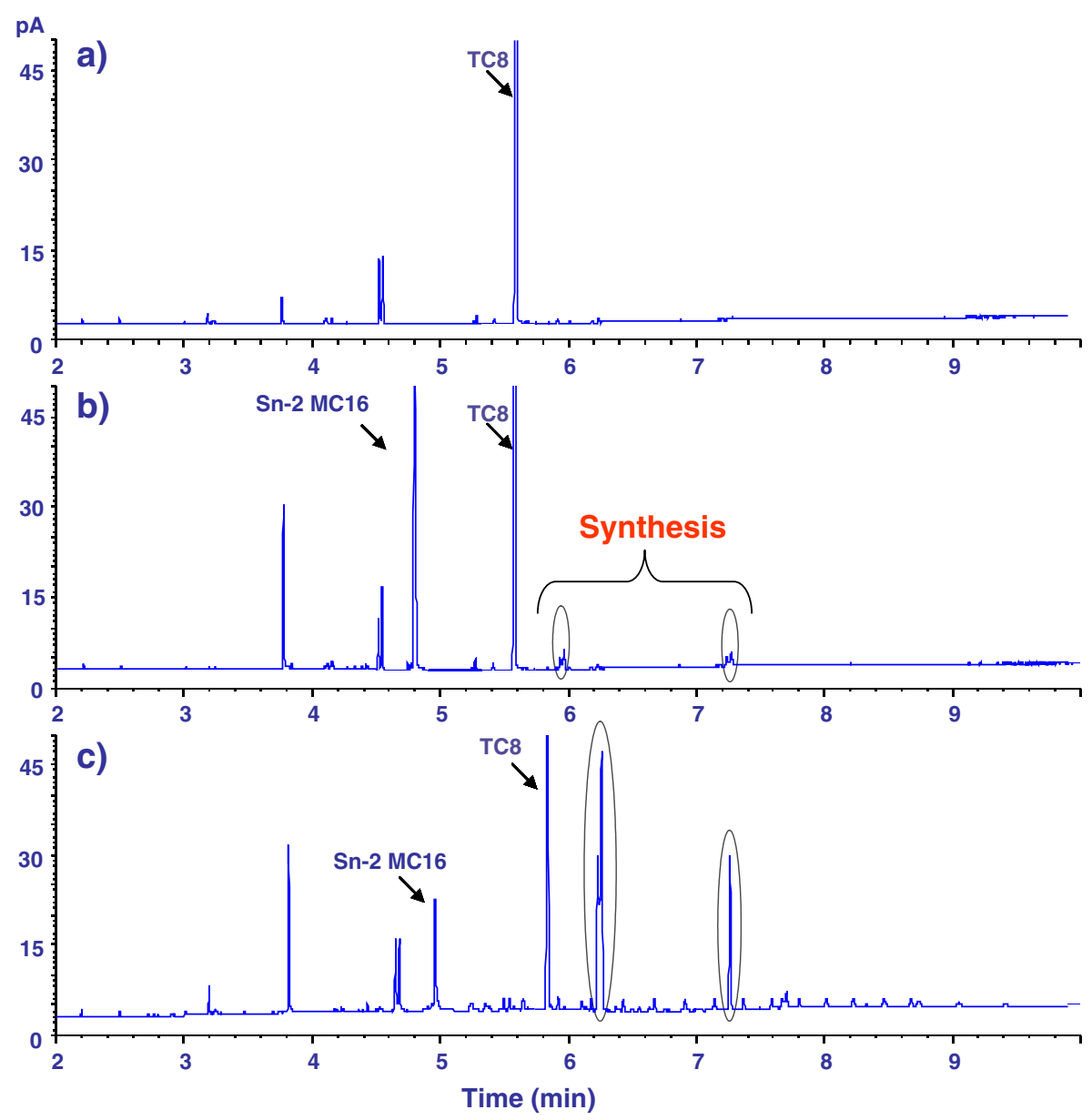

Fig. 5 GC-FID chromatogram of the oil phase from a a biphasic system consisting of $5.0 \mathrm{ml}$ decane containing $15 \mathrm{mM}$ tricaprylin (TC8) and 1.0 phosphate buffer with $3.0 \mathrm{mg} / \mathrm{ml}$ pancreatic lipase, b the same

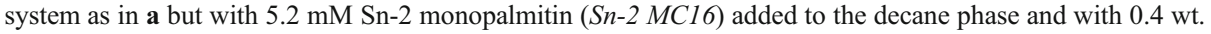
$\%$ bile salts added to the water phase, $\mathbf{c}$ the same system as in $\mathbf{b}$ but with no bile salts. Chromatograms represent the apolar phase composition after $72 \mathrm{~h}$ at $25^{\circ} \mathrm{C}$

Sn-2 position and caprylic acid in the Sn-1 and Sn-3 positions. These acyl glycerides will form by lipase-catalyzed esterification of the $\mathrm{Sn}-2$ monoglyceride by caprylic acid generated by lipase-catalyzed hydrolysis of the added tricaprylin. This type of reaction pattern is analogous to that discussed above in connection to Fig. 3.

Figure $5 \mathrm{~b}$ shows the oil phase composition of the same system as that of Fig. $5 \mathrm{c}$ after the same reaction time but with bile salts added to the water phase. Thirty seven percent of the triglyceride had been hydrolyzed, indicating a higher lipase activity in the bile-containing system, most likely due to the bile salt, allowing more space for lipase at the interface. Almost no peaks at longer reaction times than tricaprylin are present. This is probably a result of the efficient solubilization of the caprylic acid generated by lipase-catalyzed hydrolysis of tricaprylin. The solubilization leads to the removal of the caprylic acid from the reaction zone, thus preventing esterification of monopalmitin into di- and triglycerides. 
In order to assess if lipase-catalyzed synthesis in an aqueous environment is restricted to Sn-1,3 regiospecific lipases, the nonregiospecific lipase from C. rugosa was added to the same buffer/decane system with caprylic acid and tripalmitin dissolved in the oil phase. The product composition obtained after $42 \mathrm{~h}$ was compared to that obtained after the same reaction time with the $\mathrm{Sn}-1,3$ regiospecific porcine pancreas lipase. The results are shown in Fig. 6. Whereas Sn-1,3 regiospecific lipase generates Sn-2 monoglycerides and free fatty acids upon hydrolysis of tripalmitin, lipase from C. rugosa produces glycerol and palmitic acid as final products of lipolysis. Due to accumulation of noncleavable monoglycerides, pancreatic lipase can use this substrate to perform esterification with free fatty acids (either caprylic or palmitic acid). Figure $6 \mathrm{~b}$ confirms that new peaks are formed only for the enzyme that exhibits regiospecificity.

\section{Microemulsions}

Microemulsions are attractive as reaction systems for bioorganic reactions because they enable solubilization of both hydrophobic and hydrophilic molecules in a macroscopically single phase that is thermodynamically stable. Stark et al. have shown that the reaction rate and the yield of lipase-catalyzed palm oil hydrolysis depend on the nature of the surfactant
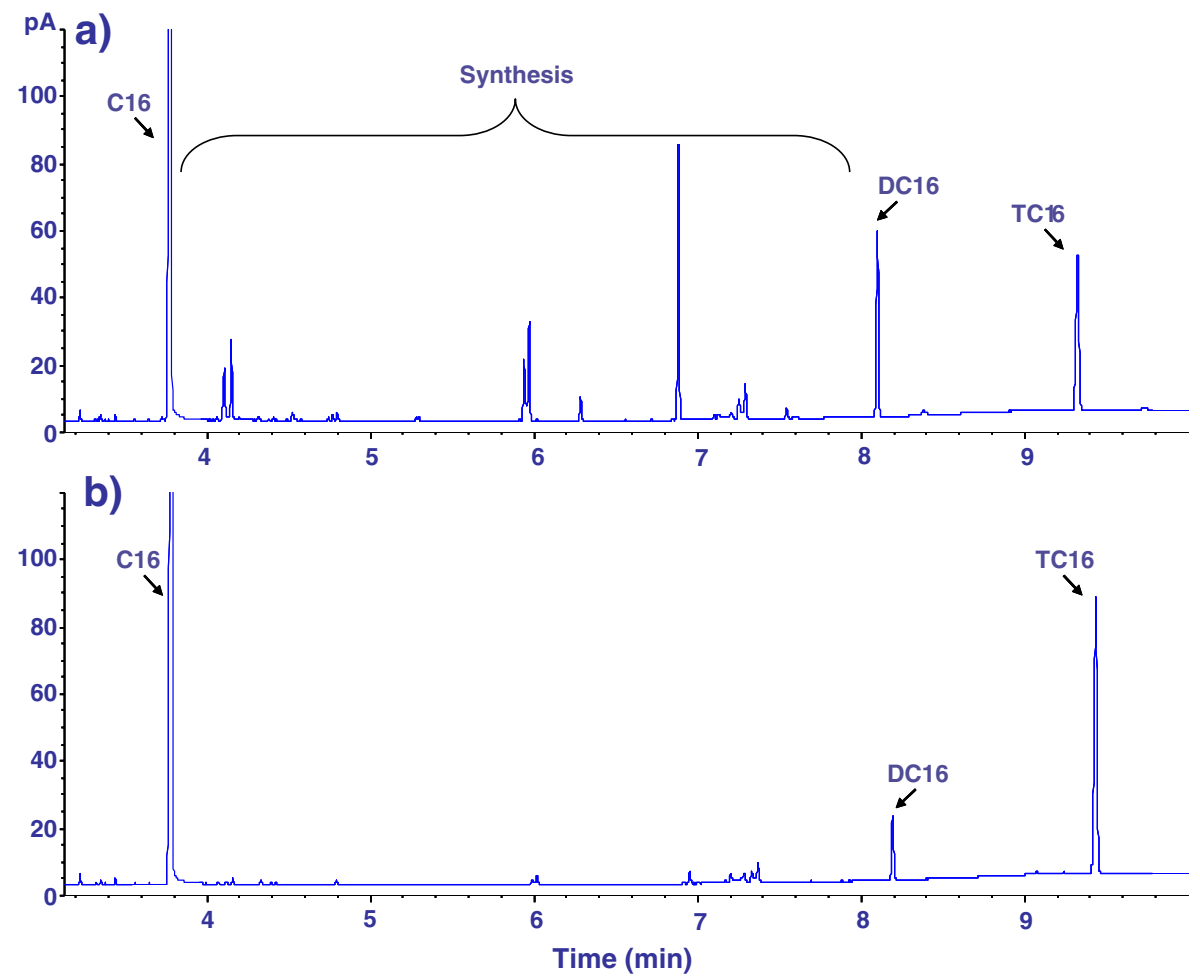

Fig. 6 GC-FID chromatogram of the oil phase of a biphasic system consisting of $3.0 \mathrm{ml}$ decane, containing $3.7 \mathrm{mM}$ tripalmitin (TC16) and $16.2 \mathrm{mM}$ caprylic acid (C8), and $3.0 \mathrm{ml} 0.1 \mathrm{M}$ phosphate buffer to which either a 7.5 E-4 M lipase from C. rugosa or b $30 \mathrm{mg} / \mathrm{ml}$ lipase from porcine pancreas had been added. The analyses were performed after $42 \mathrm{~h}$ reaction at $38^{\circ} \mathrm{C}$. DC16 and $\mathrm{C} 16$ stand for dipalmitin and palmitic acid, respectively 
used for formulating the microemulsion [35-37]. Whereas the interface of a microscopic two-phase system is static in nature, the internal interface of a microemulsion is highly dynamic, with the amphiphiles constantly moving back and forth between the interface and the bulk phases and with the whole structure disintegrating and reforming on the time-scale of milliseconds [38]. In order to investigate whether or not the high degree of dynamics will influence the activity of the lipase, reactions analogous to those performed in the biphasic system were performed in microemulsions based on different surfactants.

Microemulsions were formulated with either $\mathrm{Sn}-1 / 3$ or $\mathrm{Sn}-2$ monoglyceride as surfactant. The $\mathrm{Sn}-1,3$ regiospecific lipase from $R$. miehei was used as catalyst and pNPP was employed as a lipophilic substrate. The reaction was monitored by measuring the amount of 4-nitrophenol formed as discussed above. The same reaction was also carried out in a microemulsion based on the cationic surfactant cetyltrimethylammonium bromide (CTAB) with the water component being either distilled water or a $0.1-\mathrm{M} \mathrm{NaCl}$ solution.

Figure 7 shows the degree of conversion after $24 \mathrm{~h}$ at $25^{\circ} \mathrm{C}$. It can be seen that, with Sn-2 monopalmitin as surfactant, there is no hydrolysis of pNPP at all. Evidently, the noncleavable and very interfacially active monoglyceride prevents the enzyme from reaching the substrate that resides entirely in the apolar phase. Thus, in this respect, the internal interface of the microemulsion behaves just as the macroscopic interface of the biphasic system.

With Sn-1/3 monopalmitin as surfactant, there is a moderate conversion after $24 \mathrm{~h}$. As discussed above, Sn-1/3 monopalmitin is equally interfacially active as Sn-2 monoglyceride and, as long as it is intact, it is equally effective in expelling lipase from the interface. The $\mathrm{Sn}-1 / 3$ monopalmitin is a substrate for the Sn-1,3 regiospecific lipase, however, and is therefore gradually degraded into fatty acid and glycerol. The fatty acid formed is also amphiphilic, but it is not so interfacially active that it can expel the lipase from the reaction zone. The hydrolysis of pNPP that occurs in the Sn-1/3 monoglyceride-based microemulsion

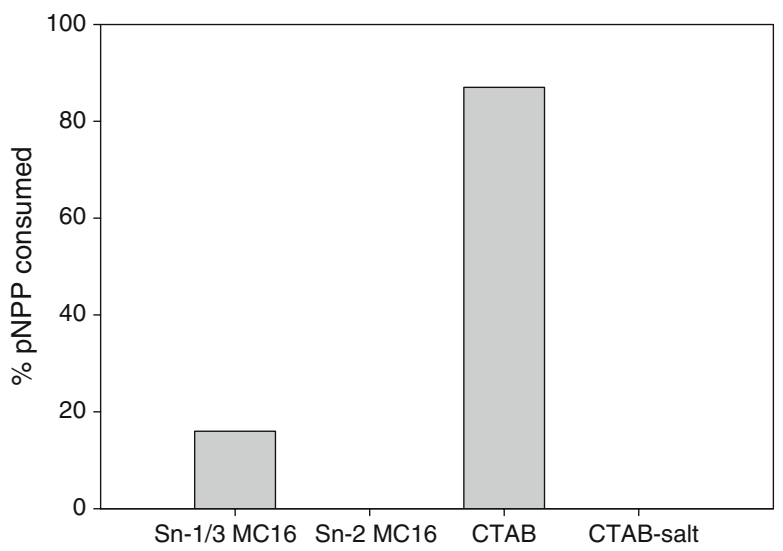

Fig. 7 Degree of conversion of 4-nitrophenylpalmitate $(p N P P)$ in a water-in-oil microemulsion containing 6.6 E-4M lipase from $R$. miehei. The microemulsion was prepared from $10 \mu$ decane, $180 \mu$ l tert-butanol, $10 \mu \mathrm{l}$ $0.1 \mathrm{M}$ phosphate buffer $\mathrm{pH}$ 7.0, and 1.22 M surfactant [either Sn-1/3 monopalmitin (Sn-1/3 MC16), Sn-2 monopalmitin (Sn-2 MC16), cetyltrimethylammonium bromide $(C T A B)$, or cetyltrimethylammonium bromide with $0.1 \mathrm{M} \mathrm{NaCl}(C T A B$-salt $)]$. The spectrophotometry measurements at $405 \mathrm{~nm}$ were performed after $24 \mathrm{~h}$ reaction at $25^{\circ} \mathrm{C}$ 
(but not in the Sn-2-based system) can most likely be attributed to an at least partial degradation of the monoglyceride.

The results with CTAB are unexpected. One may speculate that the cationic surfactant will interact with the lipase [39], which carries a net negative charge, in such a way that access of the substrate to the active site is facilitated. This is, however, contrary to previous results from studies of how different surfactants influence the activity of lipases. A cationic surfactant has been reported to retard the enzymatic activity compared to an anionic and a nonionic surfactant [40]. However, lipase biocatalysis in CTAB microemulsions has been shown to be feasible [41].

It is also highly surprising that when the reaction is carried out with CTAB as surfactant and with the water component of the microemulsion consisting of a $0.1-\mathrm{M} \mathrm{NaCl}$ solution, there is virtually no enzymatic reaction at all. This is not due to phase separation caused by the high electrolyte concentration. As can be seen from Fig. 8, the solution remains only slightly opaque during the course of the reaction. A phase separation would have been seen as an increase in turbidity.

One may speculate that the lack of reaction in the CTAB-salt system is caused by an inhibitory effect by the salt on the lipase activity. In order to test this hypothesis, the same enzymatic hydrolysis was performed in the buffer/decane biphasic system with pNPP present in the apolar phase and with the water phase consisting of either distilled water or a $0.1-\mathrm{M} \mathrm{NaCl}$ solution. Full conversion of pNPP into nitrophenol was obtained after $4 \mathrm{~h}$ for both systems. Evidently, the high salt concentration as such does not affect the enzyme's activity. Therefore, the suppression of pNPP lipolysis in a microemulsion stabilized with $\mathrm{CTAB}$ at $0.1 \mathrm{M}$ is attributed to an exclusion of the substrate from the interface. The CTAB molecular surface area is expected to be decreased at an increasing salt concentration. This phenomenon should be attributed to a screening of the cationic groups leading to a low repulsion of the polar head group. Therefore, pNPP is most likely excluded from the interface due to the high packing of CTAB molecules. Under these circumstances, lipase is unable to reach the substrate from the apolar phase.

Next, we wanted to investigate if a Sn-2 monoglyceride prevents lipolysis by a Sn-1,3 regiospecific lipase in a microemulsion as previously observed in macroscopic interfaces. A water-in-oil microemulsion was formulated with Sn-2 monopalmitin as amphiphile and

Fig. 8 Light transmission over the length of a $55-\mathrm{mm}$ test tube at different storage times at $25^{\circ} \mathrm{C}$ of a microemulsion prepared from $10 \mu \mathrm{l}$ decane, $180 \mu \mathrm{l}$ tert-butanol, $10 \mu \mathrm{l} 0.1 \mathrm{M}$ phosphate buffer $\mathrm{pH} 7.0$, and 1.22 M cetyltrimethylammonium bromide with $0.1 \mathrm{M} \mathrm{NaCl}$

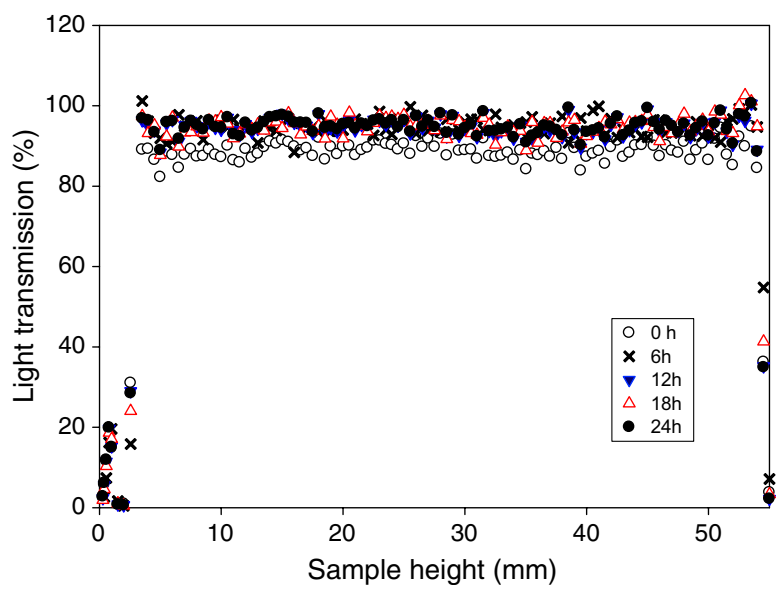


with tricaprylin dissolved in the hydrocarbon component. Lipase from $R$. miehei was added to the aqueous component. Gas chromatography results confirmed that no hydrolysis had taken place after $5 \mathrm{~h}$ at $25^{\circ} \mathrm{C}$ (data not shown). When the surfactant was changed to $\mathrm{Sn}-1 / 3$ monopalmitin, most of the triglyceride had been hydrolyzed under the same conditions.
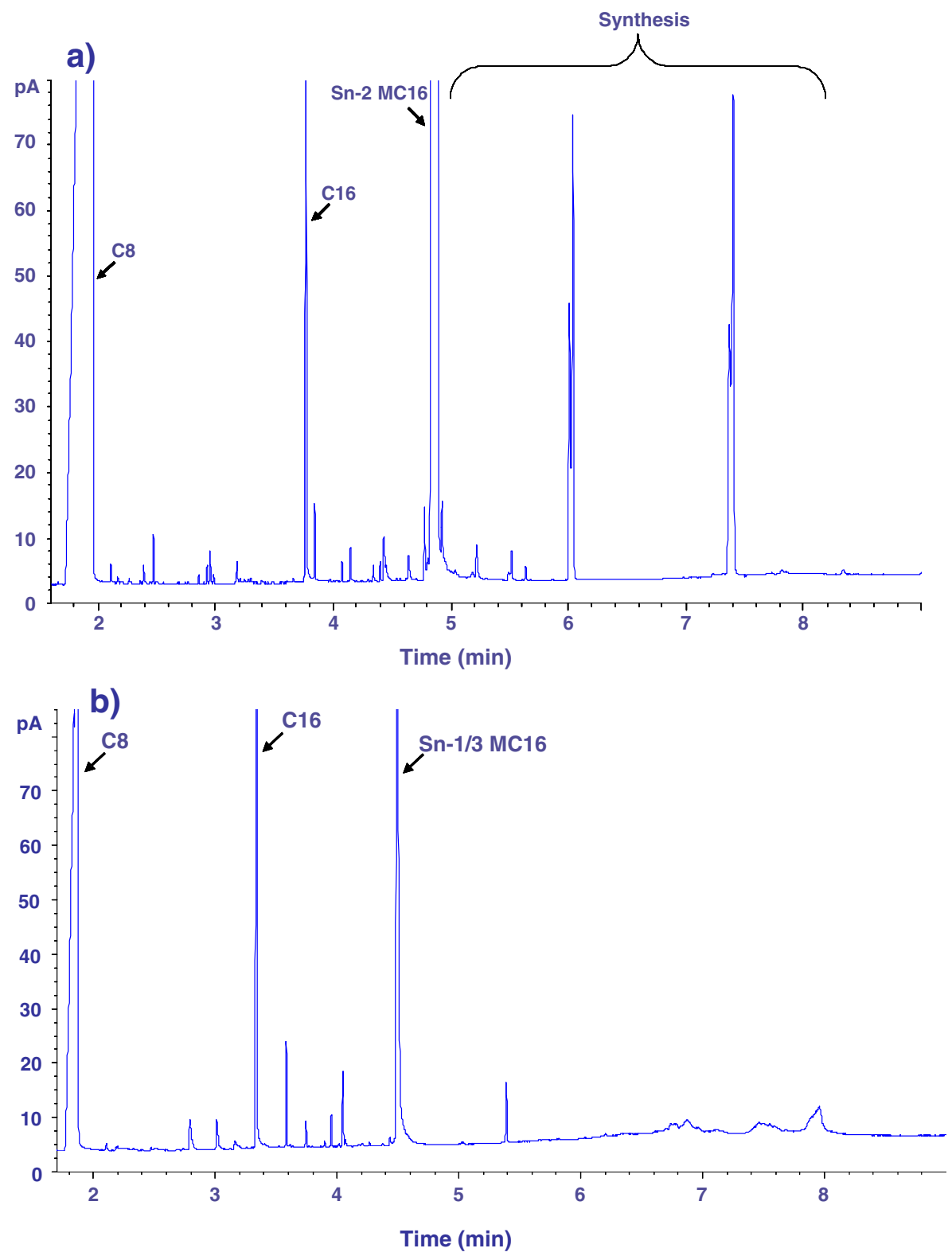

Fig. 9 GC-FID chromatogram of a water-in-oil microemulsion $20 \mu \mathrm{l}$ decane containing $0.67 \mathrm{M}$ caprylic acid (C8), $200 \mu \mathrm{l}$ tert-butanol, and $20 \mu \mathrm{l} 0.1 \mathrm{M}$ phosphate buffer $\mathrm{pH} 7.0$ and either a $0.67 \mathrm{M} \mathrm{Sn-2}$ monopalmitin (Sn-2 MC16) or $0.67 \mathrm{M} \mathrm{Sn-1/3}$ monopalmitin (Sn-1/3 MC16). The microemulsions also contained $3.3 \mathrm{E}-5 \mathrm{M}$ lipase from $R$. miehei. The analysis was performed after $88 \mathrm{~h}$ reaction at $25^{\circ} \mathrm{C}$. Palmitic acid $(C 16)$ was identified during the analysis 
These results are consistent with those obtained for the decane/buffer two-phase system. For both the macroscopic (and static) and the microscopic (and dynamic) oil-water interface, the Sn-2 monoglyceride effectively prevented access of the triglyceride to the lipase.

As discussed above (and presented in Fig. 3), a $\mathrm{Sn}-1,3$ regiospecific lipase is able to catalyze esterification of a Sn-2 monoglyceride forming di- and triglycerides with free fatty acids present in the formulation. In order to test if this is the case also in a system with a highly dynamic interface, a water-in-oil microemulsion was formulated with either Sn-2 or Sn-1/3 monopalmitin as surfactant and caprylic acid present in the hydrocarbon oil component. The Sn-1,3 regiospecific lipase from $R$. miehei was present in the formulation. Figure 9 shows the product composition for the two reactions after $88 \mathrm{~h}$ at $25^{\circ} \mathrm{C}$. It can clearly be seen that, with the Sn-2 monoglyceride as amphiphile, new molecules have been formed that are more apolar than the initial reactants. Most likely, these are the diglyceride and the triglyceride with a palmitoyl (hexadecanoyl) chain in the Sn-2 position and with capryloyl (octanoyl) chains in the Sn-1/3 position and in the Sn-1,3 positions, respectively. A peak from palmitic acid is also present in Fig. 9a. Evidently, a small fraction of the Sn-2 monopalmitin has been degraded. This may either be due to direct hydrolysis of the Sn-2 monopalmitin in spite of the enzyme being $\mathrm{Sn}-1,3$ regiospecific or to acyl group migration followed by hydrolysis of the 1/3-monopalmitin formed. The degree of hydrolysis of Sn-2 monopalmitin is low, however. According to the peak integrals, less than $5 \%$ of the monoglyceride has been converted into fatty acid.

When Sn-1/3 was used as microemulsion surfactant, the product composition was very different (Fig. 9b). Around 29\% of the monoglyceride had been converted to fatty acid and there were almost no products more apolar than the starting monoglyceride. Hence, one can conclude that no di- or triglycerides were formed.

\section{Conclusion}

The results unambiguously show that the ability of a $\mathrm{Sn}-1,3$ regiospecific lipase to catalyze hydrolysis of triglycerides is radically reduced if a short- or medium-chain Sn2 monoglyceride is present in the formulation. The lipase, which is present in a sublayer just below the oil-water interface, is capable of catalyzing esterification of the Sn-2 monoglyceride, however, leading to formation of di- and triglycerides. Analogous results were obtained with an apolar substrate, pNPP, present in the oil phase of a biphasic system.

It is interesting that the results obtained in a microemulsion based on a monoglyceride as surfactant are very similar to those obtained in a macroscopic two-phase system with the same oil and water components as in the microemulsion. This is a clear indication the degree of dynamics of the interface, which is very high for the microemulsion and extremely low for the biphasic system, is not an important parameter. The Sn-2 monoglyceride is capable of preventing the triglyceride from reaching the active site of the lipase even when the substrate and the enzyme are separated by an interface that is under constant reconstruction. Most of the intake fat is not readily bioaccessible and, therefore, has no direct caloric impact. Therefore, we anticipate that our findings might provide a new platform for the control of fat metabolism by interfacial engineering with potential applications in the biotechnology and health domains. Moreover, our results are also encouraging for lipase-catalyzed synthesis of structured lipids in aqueous solutions with defined interfacial composition. 


\section{References}

1. Mozaffar, Z., Weete, J. D., \& Dute, R. (1994). Influence of surfactants on an extracellular lipase from Pythium ultimum. Journal of the American Oil Chemists Society, 71, 75-79. doi:10.1007/BF02541475.

2. Holmberg, K., Nyden, M., Lee, L., Malmsten, M., \& Jha, B. (2000). Interactions between a lipase and charged surfactants - a comparison between bulk and interfaces. Advances in Colloid and Interface Science, 88, 223-241. doi:10.1016/S0001-8686(00)00046-4.

3. Watzke, H., Leser, M. E., \& Miller, R. (2008). Lipases at interfaces - a review. Advances in Colloids and Interface Science. doi:10.1016/j.cis.2008.06.001.

4. Furuhashi, M., Tuncman, G., Görgün, C., Makowski, L., Atsumi, G., Vaillancourt, E., et al. (2007). Treatment of diabetes and atherosclerosis by inhibiting fatty-acid-binding protein aP2. Nature, 447, 959967. doi:10.1038/nature05844.

5. Muderhwa, J., \& Brockman, H. (1992). Lateral lipid distribution is a major regulator of lipase activity. Implications for lipid-mediated signal transduction. The Journal of Biological Chemistry, 267, 2418424192.

6. Aloulou, A., Rodriguez, J., Fernandez, S., Oosterhout, D., Puccinelli, D., \& Carrière, F. (2006). Exploring the specific features of interfacial enzymology based on lipase studies. Biochimica et Biophysica Acta, 1761, 995-1013.

7. Verger, R., Pattus, F., Pieroni, G., Riviere, C., Ferrato, F., Leonardi, J., et al. (1984). Regulation by the "interfacial quality" of some biological activities. Colloids and Surfaces, 10, 163-180. doi:10.1016/ 0166-6622(84)80018-9.

8. Gargouri, Y., Julien, R., Bois, A. G., Verger, R., \& Sarda, L. (1983). Studies on the detergent inhibition of pancreatic lipase activity. Journal of Lipid Research, 24, 1336-1342.

9. Stamatis, H., Xenakis, A., \& Kolisis, F. N. (1999). Biorganic reactions in microemulsions the case of lipases. Biotechnology Advances, 17, 293-318. doi:10.1016/S0734-9750(99)00007-5.

10. Favé, G., Coste, T. C., \& Armand, M. (2004). Physicochemical properties of lipids: new strategies to manage fatty acid bioavailability. Cellular and Molecular Biology, 50, 815-831.

11. Mackie, A., Gunning, A., Ridout, M., Wilde, P., \& Morris, V. (2001). Orogenic displacement in mixed $\beta$-lactoglobulin/ $\beta$-casein films at the air/water interface. Langmuir, 17, 6593-6598. doi:10.1021/ la010687g.

12. Mackie, A., Gunning, A., Ridout, M., Wilde, P., \& Rodriguez Patino, J. (2001). In situ measurement of the displacement of protein films from the air/water interface by surfactant. Biomacromolecules, 2 , 1001-1006. doi:10.1021/bm015540i.

13. Mackie, A., Gunning, A., Pugnaloni, L., Dickinson, E., Wilde, P., \& Morris, V. (2003). The growth of surfactant domains in protein films. Langmuir, 19, 6032-6038. doi:10.1021/la034409o.

14. Ferri, J., Dong, W., Miller, R., \& Möhwald, H. (2006). Elastic moduli of asymmetric ultrathin freestanding polyelectrolyte nanocomposites. Macromolecules, 39, 1532-1537. doi:10.1021/ma0516485.

15. Fainerman, V., Leser, M., Michel, M., Lucassen-Reynders, E., \& Miller, R. (2005). Kinetics of the desorption of surfactants and proteins from adsorption layers at the solution/air interface. The Journal of Physical Chemistry B, 109, 9672-9677. doi:10.1021/jp050212o.

16. Grigoriev, D., Leser, M., Michel, M., \& Miller, R. (2007). Mixed micelles as delivery systems for enhanced emulsifier adsorption at the air/water interface: Sodium stearoyl lactylate (SSL)/Tween80 solutions. Colloids and Surfaces A: Physicochemical and Engineering Aspects, 301, 158-165. doi:10.1016/j.colsurfa.2006.12.048.

17. Mackie, A., \& Wilde, P. (2005). The role of interactions in defining the structure of mixed proteinsurfactant interfaces. Advances in Colloid and Interface Science, 117, 3-13. doi:10.1016/j. cis.2005.04.002.

18. Aksenenko, E., Kovalchuk, V., Fainerman, V., \& Miller, R. (2006). Surface dilational rheology of mixed adsorption layers at liquid interfaces. Advances in Colloid and Interface Science, 122, 57-66. doi:10.1016/j.cis.2006.06.012.

19. Grigoriev, D., Derkatch, S., Krägel, J., \& Miller, R. (2007). Relationship between structure and rheological properties of mixed BSA/Tween 80 adsorption layers at the air/water interface. Food Hydrocolloids, 21, 823-830. doi:10.1016/j.foodhyd.2006.08.018.

20. Turro, N., Lei, X., Ananthapadmanabhan, K., \& Aronson, M. (1995). Spectroscopic probe analysis of protein-surfactant interactions: The BSNSDS system. Langmuir, 11, 2525-2533. doi:10.1021/ la00007a035.

21. Wüstneck, R., Krägel, J., Miller, R., Wilde, P., \& Clark, D. (1996). The adsorption of surface-active complexes between $\beta$-casein, $\beta$-lactoglobulin and ionic surfactants and their shear rheological behaviour. Colloids and Surfaces A: Physicochemical and Engineering Aspects, 114, 255-265. doi:10.1016/09277757(96)03535-2. 
22. Dickinson, E. (1999). Adsorbed protein layers at fluid interfaces: interactions, structure and surface rheology. Colloids and Surfaces B, 15, 161-176.

23. Miller, R., Fainerman, V., Makievski, A., Krägel, J., Grigoriev, D., Kazakov, V., et al. (2000). Dynamics of protein and mixed protein/surfactant adsorption layers at the water/fluid interface. Advances in Colloid and Interface Science, 86, 39-82. doi:10.1016/S0001-8686(00)00032-4.

24. Krägel, J., O’Neill, M., Makievski, A., Michel, M., Leser, M., \& Miller, R. (2003). Dynamics of mixed protein-surfactant layers adsorbed at the water/air and water/oil interface. Colloids and Surfaces B, 31, $107-114$

25. Miller, R., Leser, M., Michel, M., \& Fainerman, V. (2005). Surface dilational rheology of mixed $\beta$ lactoglobulin/surfactant layers at the air/water interface. The Journal of Physical Chemistry B, 109, 13327-13331. doi:10.1021/jp0510589.

26. Valivety, R., Halling, P., \& Macrae, A. (1993). Water as a competitive inhibitor of lipase-catalysed esterification in organic media. Biotechnology Letters, 15, 1133-1138. doi:10.1007/BF00131203.

27. O'Connor, C., \& Walde, P. (1986). Interactions of human milk lipase with sodium taurocholate and other surfactants. Langmuir, 2, 139-146. doi:10.1021/la00068a004.

28. Rotenberg, Y., Boruvka, L., \& Neumann, A. W. (1983). Determination of surface tension and contact angle from the shapes of axisymmetric fluid interfaces. Journal of Colloid and Interface Science, 93, 169-183. doi:10.1016/0021-9797(83)90396-X.

29. Reis, P., Holmberg, K., Miller, R., Krägel, J., Grigoriev, D. O., Leser, M. E., et al. (2008). Competition between lipases and monoglycerides at interfaces. Langmuir, 24, 7400-7407. doi:10.1021/la800531y.

30. Reis, P., Miller, R., Leser, M. E., Watzke, H., Fainerman, V. B., \& Holmberg, K. (2008). Adsorption of polar lipids at the water-oil interface. Langmuir, 24, 5781-5786. doi:10.1021/la704043g.

31. Reis, P., Holmberg, K., Miller, R., Leser, M. E., Raab, T., \& Watzke, H. (2008). Lipase reaction at interfaces as self-limiting processes. Comptes Rendus, in press.

32. Reis, P., Raab, T., Chuat, J. Y., Leser, M. E., Miller, R., Watzke, H., et al. (2008). Influence of surfactants on lipase fat digestion in a model gastro-intestinal system. Food Biophysics, doi:10.1007/s11483-0089091-6.

33. Jaye, M., Lynch, K., Krawiec, J., Marchadier, D., Maugeais, C., Doan, K., et al. (1999). A novel endothelial-derived lipase that modulates HDL metabolism. Nature Genetics, 21, 424-428. doi:10.1038/ 7766.

34. Hofman, A. F. (1963). The function of bile salts in fat absorption. The solvent properties of dilute micellar solutions of conjugated bile salts. The Biochemical Journal, 89, 57-68.

35. Stark, M. B., Skagerlin, P., Holmberg, K., \& Carlfors, J. (1990). Dependence of the activity of a rhizopus lipase on microemulsion composition. Colloid \& Polymer Science, 268, 384-388. doi:10.1007/ BF01411681.

36. Skagerlind, P., Jansson, M., Hult, K. (1992). Surfactant interference on lipase catalysed reactions in microemulsions. Journal of Chemical Technology \& Biotechnology, 54, 277-282.

37. Holmberg, K., \& Osterberg, E. (1988). Enzymatic preparation of monoglycerides in microemulsion. Journal of the American Oil Chemists' Society, 65, 1544-1548. doi:10.1007/BF02898325.

38. Fletcher, P. D., \& Horsup, D. (1992). Droplet dynamics in water-in-oil microemulsions and macroemulsions stabilised by non-ionic surfactants. Correlation of measured rates with monolayer bending elasticity. Journal of the Chemical Society. Faraday Transactions, 88, 855-864. doi:10.1039/ $\mathrm{ft} 9928800855$.

39. Skagerlind, P., Jansson, M., Bergenstahl, B., \& Hult, K. (1995). Binding of Rhizomucor miehei lipase to emulsion interfaces and its interference with surfactants. Colloids and Surfaces B, 4, 129-135. doi:10.1016/0927-7765(94)01167-4.

40. Skagerlind, P., \& Holmberg, K. (1994). Effect of the surfactant on enzymatic hydrolysis of palm oil in microemulsions. Journal of Dispersion Science and Technology, 15, 317-322. doi:10.1080/ 01932699408943560.

41. Rees, G. D., \& Robinson, B. H. (2004). Esterification reactions catalyzed by Chromobacterium viscosum lipase in CTAB-based microemulsion systems. Biotechnology and Bioengineering, 45, 344-355. doi:10.1002/bit.260450409. 